\title{
Implant and patient tracking: the opinion of a patient
}

\author{
Jan J. Nouwen \\ Stichting Patientenbelangen Orthopaedie, clo NL Pharma B.V., P.O. Box 30068, \\ 1303 AB Almere, The Netherlands
}

A paper such as this deserves to begin with a personal word of introduction. Although a lawyer by education, I have during the last twenty-five years of active career been a newspaper publisher. In that capacity I have engaged very fully in the close international collaboration which exists between newspaper publishers in all parts of the world, always participating in the annual world newspaper congresses. They are important gatherings, with everyone present who plays a role in newspaper publishing, to say nothing of the heads of state and politicians of the host countries. Everyone, that is, except one vital category: the newspaper reader. I have never met a reader, nor yet a representative of the reader, at any of these meetings.

At this medical congress you have done better; you have at least one patient present - and that is myself. Perhaps, then, I have a certain right to your attention. Fortunately, I am not speaking merely on my own behalf; I am speaking on behalf of the organization which represents orthopaedic patients here in The Netherlands. Founded some five years ago, at the initiative of the Amsterdam orthopaedic surgeon Prof. Marti, its main activities were in the beginning the answering of questions from orthopaedic patients, naturally in a manner which did not infringe upon medical privilege, and the twice-yearly publication of a patient information journal with a circulation of sixty thousand.

Two years ago we developed a policy plan. It was decided that one of our major priorities must be to conduct a survey into the bottlenecks standing in the way of orthopaedic care in this country, and into the social relevance of orthopaedic care. There were good reasons for this choice. In The Netherlands, patients unfortunately often have to cope with relatively long waiting lists for orthopaedic treatment, naturally with regional and individual variations, and suspect that these waiting lists are often longer than those for other forms of specialized medical treatment. The goals of this survey are firstly to determine where in the orthopaedic field in our country bottlenecks exist, with the result that a patient may not receive the right medical treatment in good time, or without incurring greater expense than necessary, or at all. Secondly, we are seeking to determine the social relevance of orthopaedic care, and especially the increased costs of providing social security benefits (or the greater loss in labour productivity) which Dutch society has to bear as a result of this less than optimal treatment of many patients. Thirdly, we have set out to forecast the influence of demographic developments on the future level of need for orthopaedic care. Fourthly, we want to identify those circumstances of developments which could guide our activities for the coming years in attempting to improve the conditions under which orthopaedic care is made available in this country. 
Under the auspices of the Institute for Medical Technology of the Erasmus University, Rotterdam, a first survey of this type was initiated at the request of our Foundation by Mrs. Joosten-van Zwanenberg as a dissertation study, and is now nearing its completion. It appears already that in the course of this survey quite a number of important data have been collected, on the basis of which proposals can be formulated to improve the conditions under which orthopaedic care in this country is provided. We will elaborate on these results in the coming months and will try to draw up a number of proposals or, indeed demands, to be addressed to the health authorities in the country with a view to improving the conditions of orthopaedic care.

What I want to emphasize at this moment is that the Netherlands Orthopaedic Society has cooperated in this study to a major extent by helping in the collection of a number of data; in that connection let me stress that relations between our Foundation and the Orthopaedic Society are excellent. We have made it very clear that we seek to be an independent patient organization and reserve our right to formulate any points of view which we believe to be correct and useful; at the same time, however, we choose to maintain the best possible relationship with the Netherlands orthopaedic specialists and their Society. I am happy to say that all this has worked out very well. Of course we shall consult the Orthopaedics Society in further elaborating and presenting the results of the survey which I have just outlined.

This present congress is devoted to the post marketing surveillance of medical implants and I have been invited to present the point of view of a patient. I can, in fact, do that in relatively few words. As a patient organization we are very strongly in favour of the activities which you are discussing. The registration and monitoring of medical implants can improve the quality and safety both of the products themselves and of the patient care in which they are used. The result obtained can clearly enhance scientific research into implants and their quality, and they will be of great importance to the individual patient checking whether an implant is functioning as it should, in understanding and solving problems which he or she experiences, and in correctly renewing the implant when the time arrives to do so.

One of the people who in this country took the initiative to develop a comprehensive system of registration for individual medical implants is Prof. Slooff from Nijmegen. Some two years ago he sought the opinion of our organization and, in so far as practical, its assistance. In the light of this request our Foundation formulated its position in this matter and reported back both to Prof. Slooff and naturally as well to the Netherlands Orthopaedic Society. First of all we declared that our Foundation was in favour of complete registration of medical implants in this country and, I may add, in Europe as a whole, since such registration carries scientific weight and is in the common interest especially the interest of current and future orthopaedic patients. We further lent support to the view that the registration be entrusted to the S.I.G. institute, a national institute dealing with data on medical care. At the present time the registration system operates on a purely voluntary basis but I have been informed that more than fifty percent of Dutch orthopaedic surgeons already cooperate with the system and that happily this percentage is on the increase. It is, I understand, possible that in the future the participation of orthopaedic surgeons will be made compulsory. If this proves to be a necessry step we shall as a patient organization lend support to it, but as long as it is not necessary we shall not press for it. The imposition of legal obligation should surely be avoided where it is not strictly necessary, particularly in the field of medical care in which governments already choose to regulate - or find themselves forced to regulate - on more matters than one would in fact wish. In principle, we prefer to place our trust in the sense of responsibility and professionality of the orthopaedic surgeons in this country. 
We also commented on the question as to whether patients who are fitted with implant could themselves report the fact to the registration body, since there is as yet no complete registration system. Here we are not so sure. The goal for complete registration will surely be attained much more rapidly via the orthopaedic surgeon than through the patient. Often patients will not have all the relevant data at their disposal and some of them will either not be inclined to cooperate in the registration process or be unable to do so. Acceptance by the orthopaedic surgeon of an obligation to cooperation in the registration implants is thinkable and feasible, an obligation lodged with the patient is not. To impose such a duty on the latter would be a breach of his personal freedom as a patient - a matter which, as you know, has been the subject of legal protection in this country from as recently as the beginning of April 1995. This of course does not detract from the desirability of patients cooperating to the best of their ability; our Foundation, seeking to act as the patient representative, will provide all the cooperation which is requested of us so long as patients themselves are not emburdened with a legal duty which they cannot reasonably be expected to execute.

Finally, on the subject of a patient identification card, which was raised earlier at this meeting, our Foundation has not yet formulated its opinion. It shall do so in the near future and personally I expect the outcome to be positive. A patient carrying such a card, containing his medical data, can rapidly be identified and the essential information in his case will be available immediately, thus helping to avoid both medical and administrative error. Such a card will also promote the exchange of information between the medical expert involved in his treatment as well as the hospitals and other institutions with which he may be in contact. I hope and expect that the medical profession will increasingly exploit the possibilities offered by modern information and communication technology, including the opportunities opened up by the so-called electronic information highway. Since, as I have pointed out, patients' rights in this country are also protected by a law on the registration of the individual, no problems need arise in this respect. Naturally one can never impose an obligation on a patient to carry an information card or even cooperate, since that would run counter to his personal freedom, but in fact one can reasonably assume that every patient will cooperate freely by carrying the card since this will be in his or her direct personal interest. I anticipate that our Foundation will, when asked for its opinion on this matter by individual patients, advise them accordingly.

The only question which comes to mind in connection with patient identity cards is whether the registration system involving such cards should be in the hands of a commercial supplier - reliable though the supplier is in this case. I hasten to emphasize that our Foundation has a very good relationship indeed with Ortomed. The firm has an excellent reputation in our orthopaedic world. The question is simply whether it would not in principle be more proper to entrust registration to an independent medical care institute, so that not even the suggestion can arise that too much information is being entrusted to a single interested party. There must at all events be a guarantee that the information will be available to all who have a legitimate interest in its use, in the interest either of individual patients or of medical science, at no more than actual cost. This does not detract in any way from my positive attitude - which I anticipate will be parallelled by the views of our Foundation - towards your efforts to develop the availability and accessibility of patient information. 